\title{
Change management: pourquoi changer et pour quoi changer
}

\author{
Anne-Geneviève Bütikofer
}

lic. iur., secrétaire générale de la FMH

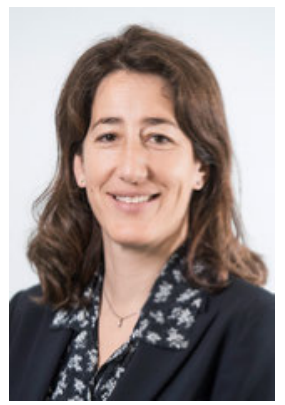

Chaque entreprise, chaque organisation traverse des périodes de changement, qu'elles soient petites ou grandes, publiques ou privées, familiales ou institutionnelles. Ainsi ces propos s'adressent aussi bien aux pratiques privées, aux cabinets de groupes, aux institutions qu'au Secrétariat général (SG) de la FMH, bref à tout lecteur. Le Change management est aujourd'hui partout présent dans notre quotidien professionnel. Il entre en jeu que nous soyons concernés par un renouvellement des lieux de travail ou des outils de travail, l'introduction de nouveaux modes de collaboration (par ex. par la digitalisation), la mise sur pied de mesures de bien-être au travail, le renforcement des compétences de management ou encore la valorisation et la reconnaissance des compétences.

Le processus constant et souple du Change management doit permettre de mieux s'épanouir dans le travail tout en répondant aux attentes.

L'efficacité et la performance de nos structures dépendent de notre capacité à mettre en place les transformations sans qu'il n'y ait d'impact défavorable sur les collaborateurs respectivement sur le travail, mais bien au contraire que cela se traduise par un impact positif. Car le Change management touche avant tout à l'être humain, mais surtout repose sur lui pour être couronné de succès. Procéder à des changements exige donc un accompagnement étroit des personnes concernées afin de faciliter leur adaptation. Sans explication, communication, soutien et formation, le processus de changement sera voué à l'échec car les collaborateurs ne s'identifieront pas aux nouveaux processus et auront du mal à s'impliquer. Alors que le domaine de la santé est soumis à de nombreuses pressions, que les conditions économiques sont tendues et que les clients sont de plus en plus avisés (patients pour les médecins, ou membres/clients pour le SG de la FMH), le Change management doit être un processus constant et souple, qui permet à nos équipes de s'en nourrir pour mieux s'épanouir dans

leur travail et répondre aux attentes à leur égard. En ce qui concerne le SG de la FMH, qui fait face de manière permanente au besoin de changement et d'adaptation, si la direction est responsable de la mise en place d'une bonne gestion du changement, elle doit être appuyée à la fois par les organes, respectivement le comité central et la chambre médicale, mais aussi à l'interne par les cadres sur lesquels une importante part du succès repose car ils connaissent le mieux les collaborateurs et sauront identifier les risques liés au changement y compris la "résistance au changement» et y pallier, mais également la communication interne, les RH et les personnes chargées des systèmes d'information respectivement de la transition numérique. En effet, les outils numériques apportent un soutien non négligeable à la gestion du changement, mais ce pour autant qu'ils reposent sur une stratégie bien définie. A l'heure actuelle si nous souhaitons progresser nous devons mettre en place un environnement de travail plus ouvert et flexible où les employés jouissent de plus de libertés et d'autonomie. Pour être efficace nous devons introduire davantage de transparence et de collaboration avec nos collaborateurs au travers d'un management centré sur les besoins humains, nous assurer que les nouvelles technologies nous facilitent la communication et la créativité mais également renforcer le partage des valeurs, la confiance et le bien-être. Ceci nous assurera une plus grande adaptation au

\section{Introduire davantage de transparence et} de collaboration avec nos collaborateurs mais également renforcer le partage des valeurs, la confiance et le bien-être.

changement, du plaisir au travail et donc une efficacité redoublée. Voici le menu qui attend le SG de la FMH en 2018, et je me réjouis de pouvoir le partager avec les collègues du SG de la FMH, dont l'engagement a été à nouveau exemplaire en 2017. Je vous souhaite de belles fêtes de fin d'année et que la nouvelle année vous apporte à tous son lot de changements positifs. 